\title{
Spotlight on Ki67 as a prognostic marker in early breast cancer: all that glitters may not be gold
}

\author{
Roberta Maltoni, Michela Palleschi, Sara Ravaioli* (D, Maria Maddalena Tumedei, Mattia Altini and Sara Bravaccini
}

\begin{abstract}
Kang and colleagues evaluated on a case series of 1848 breast cancer (BC) patients operated for a first primary ER positive HER2 negative BC if Ki67 expression is a significant prognostic factor only when PgR expression is low. The authors concluded that Ki67 with 10\% cut off value is a prognostic factor only under low PgR expression level in early BC. We would like to underline, that as already stated in our previous papers, we believe that proliferation is important to define the decision-making of adjuvant therapies in early BC. The issue on Ki67 detection is the poor reproducibility due to different antibody clones, platforms and scoring methods. Not less important is that different Ki67 cut off values have been used by San Gallen guidelines and changed overtime. Then, despite the interesting results, we believe it would be better to use Ki67 biomarker in according to the standard Ki67 cut off according to San Gallen guidelines. Nowadays, standardization and optimization of Ki67 is still an urgent need.
\end{abstract}

Keywords: Ki67, PgR, Breast cancer

Ki67 value has been recognized in early breast cancer (BC) but controversy still exists. We congratulate with Kang and colleagues for the very interesting paper. The authors evaluated in $1848 \mathrm{BC}$ patients operated on for a first primary Estrogen Receptor (ER) positive HER2 negative BC if Ki67 expression is a significant prognostic factor only when Progesterone Receptor (PgR) expression is low [1]. The authors concluded that Ki67 with $10 \%$ cut off value is a prognostic factor only under low PgR expression level in early BC. PgR should be considered in evaluating the prognosis of $\mathrm{BC}$ patients using Ki67 expression.

About 30 years ago, our group started a study in attempt to establish the use of chemotherapy or nothing in node negative $\mathrm{BC}$ with high tumor labelling index [2]. In 2008, our research group published the results from this phase 3 randomized multicenter study on the effects

\footnotetext{
* Correspondence: sara.ravaioli@irst.emr.it

Istituto Scientifico Romagnolo per lo Studio e la Cura dei Tumori (IRST) IRCC S, Via P. Maroncelli 40, 47014 Meldola (FC), Italy
}

of adjuvant Cyclophosphamide, Methotrexate, Fluorouracil (CMF) in patients with node-negative, rapidly proliferating $\mathrm{BC}$ [2]. The results from twelve-year subgroup analysis showed that CMF produced a 25 and $20 \%$ relative reduction in relapse and death cumulative incidence, respectively.

We recently demonstrated that PgR is an independent prognostic marker in rapidly proliferating hormone receptor positive early BC [3]. We would like to underline that, as already stated in our previous papers, we believe that proliferation is important to define the decisionmaking of adjuvant therapies in early BC [2].

The issue on Ki67 detection is the poor reproducibility due to different antibody clones, platforms and scoring methods used among the different laboratories and observers as reported by the studies on biomarkers comparison among the different centers [4]. As we already observed in our laboratory its detection by immunohistochemistry is affected by the preanalytical phase in terms of tissue over and under-fixation or delay in 


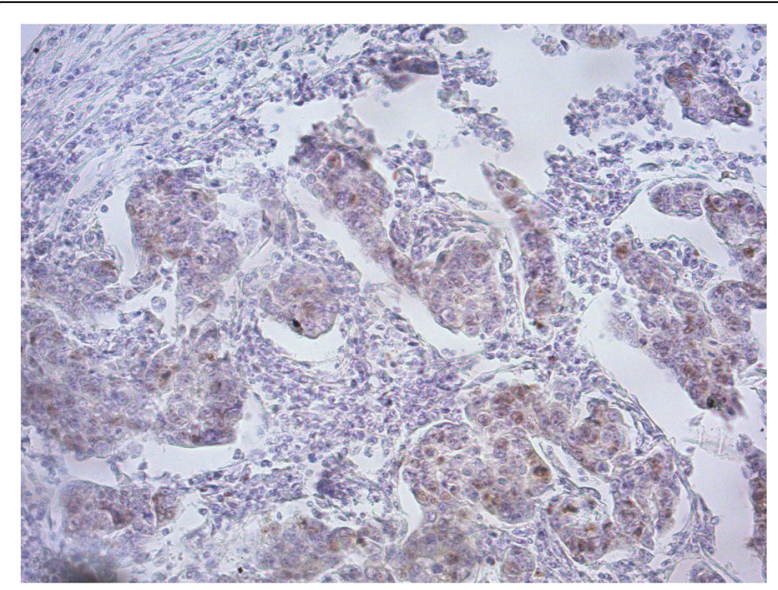

Fig. 1 Invasive breast cancer tissue with non-specific and inhomogeneous expression of Ki67 by immunohistochemistry (20X magnification)

fixation (Fig. 1) [5]. All this factors affect reproducibility of the different studies [4]. Not less important is that different Ki67 cut off values have been used by San Gallen guidelines and changed overtime from 14 to $30 \%$. Then, despite the interesting results, we believe it would be better to use Ki67 biomarker in according to the standard Ki67 cut off according to San Gallen guidelines. Nowadays, standardization and optimization of Ki67 is still a utopia and further efforts are required.

\section{Abbreviations}

BC: Breast cancer; ER: Estrogen Receptor; PgR: Progesterone Receptor; CMF: Cyclophosphamide, Methotrexate, Fluorouracil

\section{Acknowledgements}

Not applicable.

\section{Authors' contributions}

RM, MP, MA and SB analyzed and interpreted the patient data. SR and MMT performed immunohistochemical analyses. RM and SB were major contributors in writing the manuscript. All authors read and approved the final manuscript.

\section{Funding}

none.

\section{Availability of data and materials}

Data sharing is not applicable to this article as no datasets were generated or analyzed during the current study.

\section{Ethics approval and consent to participate}

The study was conducted in accordance with the Declaration of Helsinki Ethical Principles and was approved by the local ethics committee of Area Vasta Romagna (approval number 3692).

\section{Consent for publication}

All the authors give the consent for information included in the Commentary to be published in Diagnostic Pathology. I understand that the text and any pictures or videos published in the article will be freely available on the internet and may be seen by the general public. The pictures, videos and text may also appear on other websites or in print, may be translated into other languages or used for commercial purposes. I have been offered the opportunity to read the manuscript. Signing this consent form does not remove my rights to privacy.

Author name....SARA RAVAIOLI.

Date.........23/07/2020.

Signed.....Sara Ravaioli, corresponding author.

\section{Competing interests}

The authors declare that they have no competing interests.

Received: 23 July 2020 Accepted: 3 September 2020

Published online: 11 September 2020

\section{References}

1. Kang YJ, Lee HB, Kim YG, Han J, Kim Y, Yoo TK, et al. Ki-67 expression is a significant prognostic factor only when progesterone receptor expression is low in estrogen receptor-positive and HER2-negative early breast Cancer. J Oncol. 2019;7386734.

2. Amadori D, Nanni O, Volpi A, Casadei Giunchi D, Marangolo M, Livi L, et al. Phase III randomized multicenter study on the effects of adjuvant CMF in patients with node-negative, rapidly proliferating breast cancer: twelve-year results and retrospective subgroup analysis. Breast Cancer Res Treat. 2008; 108(2):259-64.

3. Bravaccini S, Bronte G, Scarpi E, Ravaioli S, Maltoni R, Mangia A, et al. The impact of progesterone receptor expression on prognosis of patients with rapidly proliferating, hormone receptor-positive early breast cancer: a post hoc analysis of the IBIS 3 trial. Ther Adv Med Oncol. 2020;12: 1758835919888999

4. Polley MY, Leung SC, Gao D, Mastropasqua MG, Zabaglo LA, Bartlett JM, et al. An international study to increase concordance in Ki67 scoring. Mod Pathol. 2015;28:778-86.

5. Amadori D, Serra P, Bravaccini S, Farolfi A, Puccetti M, Carretta E, et al. Differences in biological features of breast cancer between Caucasian (Italian) and African (Tanzanian) populations. Breast Cancer Res Treat. 2014; $145: 177-83$

\section{Publisher's Note}

Springer Nature remains neutral with regard to jurisdictional claims in published maps and institutional affiliations. 